\title{
WEAK GIBBS MEASURES AND EQUILIBRIUM STATES
}

\author{
MARIA CARVALHO AND SEBASTIÁN A. PÉREZ
}

\begin{abstract}
Aвstract. We present a simple proof of the fairly general fact that an invariant right-weak Gibbs probability measure is an equilibrium state, using a generalization of the formula of Brin and Katok for the local metric entropy. This new formula also allows us to clarify the role of the topological pressure on the standard definition of the Gibbs property.
\end{abstract}

\section{INTRODUCTION}

Let $(X, d)$ be a compact metric space, $f: X \rightarrow X$ a continuous transformation and $\varphi: X \rightarrow \mathbb{R}$ a continuous potential. The topological pressure $P_{\text {top }}(f, \varphi)$ of $f$ and $\varphi$ is a topological invariant that generalizes the notion of topological entropy of $f$, we denote by $h_{\text {top }}(f)$, in the sense that $P_{\text {top }}(f, 0)=h_{\text {top }}(f)$. A Borel $f$-invariant probability measure $\mu$ is said to be an equilibrium state for $f$ and the potential $\varphi$ if

$$
P_{\mu}(f, \varphi)=\sup _{\left\{v: f_{*}(v)=v\right\}} P_{v}(f, \varphi)
$$

where $P_{v}(f, \varphi)$ stands for the sum $h_{v}(f)+\int \varphi d v$ and the supremum is taken over all the Borel $f$-invariant probability measures. According to the Variational Principle ([9, Theorem 9.10]), the previous least upper bound coincides with the supremum evaluated on the set of ergodic probability measures, and is equal to $P_{\text {top }}(f, \varphi)$. We refer the reader to [9] for precise definitions and proofs of these results.

Given $\gamma \in \mathbb{R}$ and a continuous potential $\varphi$, we say that a Borel probability measure $\mu$ is $(\gamma, \varphi)$-weak Gibbs for the dynamical system $f$ if there exist an $\varepsilon_{0}>0$ and a subset $\Lambda \subset X$ with full $\mu$-measure such that, for every $0<\varepsilon<\varepsilon_{0}$ and every $x \in \Lambda$, there is a sequence of positive constants $\left(\delta_{n}(\varepsilon, x)\right)_{n \in \mathbb{N}}$ satisfying

$$
\lim _{\varepsilon \rightarrow 0^{+}} \limsup _{n \rightarrow+\infty} \frac{\log \delta_{n}(\varepsilon, x)}{n}=0
$$

Date: February 13, 2020.

2010 Mathematics Subject Classification. 28D05, 28D20, 37D35.

Key words and phrases. Weak Gibbs measure; Topological and metric pressure; Equilibrium state.

MC and SP were partially supported by CMUP (UID/MAT/00144/2019), which is funded by FCT with national (MCTES) and European structural funds through the programs FEDER, under the partnership agreement PT2020. SP also acknowledges financial support from a postdoctoral grant of the project PTDC/MAT-CAL/3884/2014. The authors are grateful to Artur Óscar Lopes for sharing with them his insight about non-invariant weak Gibbs measures. 
and, for every $n \in \mathbb{N}$ (or for every $n$ large enough),

$$
\delta_{n}(\varepsilon, x)^{-1} \leqslant \frac{\mu\left(B_{n}^{f}(x, \varepsilon)\right)}{\exp \left(-n \gamma+S_{n}^{f} \varphi(x)\right)} \leqslant \delta_{n}(\varepsilon, x)
$$

where

$$
B_{n}^{f}(x, \varepsilon)=\left\{y \in X: d\left(f^{i}(x), f^{i}(y)\right)<\varepsilon, \quad \forall 0 \leqslant i \leqslant n-1\right\}
$$

is the $n$th dynamical ball of $f$ at $x$ with radius $\varepsilon$. We note that the assumption (2) obliges the maps $\delta_{n}$ to satisfy $\delta_{n}(\varepsilon, x) \geqslant 1$ for every $n, x, \varepsilon$, and therefore the equality (1) implies that

$$
\lim _{\varepsilon \rightarrow 0^{+}} \liminf _{n \rightarrow+\infty} \frac{\log \delta_{n}(\varepsilon, x)}{n}=0 .
$$

In case $\mu$ satisfies only the right inequality of the condition (2), then $\mu$ is said to be $(\gamma, \varphi)$ right-weak Gibbs. One defines $(\gamma, \varphi)$-left-weak Gibbs measure similarly. A $(\gamma, \varphi)$-weak Gibbs measure is called $\varphi$-weak Gibbs if $\gamma=P_{\text {top }}(f, \varphi)$. Whenever $\Lambda=X$ and instead of (1) one has

$$
\lim _{\varepsilon \rightarrow 0^{+}} \limsup _{n \rightarrow+\infty} \sup _{x \in X} \frac{\log \delta_{n}(\varepsilon, x)}{n}=0
$$

then $\mu$ is said to be $(\gamma, \varphi)$-quasi Gibbs. Finally, $\mu$ is called a $\varphi$-Gibbs measure if it is $\varphi$-weak Gibbs on $\Lambda=X$ and each map $(\varepsilon, x) \mapsto \delta_{n}(\varepsilon, x)$ remains unchanged when $x$ varies, depending only on $\varepsilon$ (as happens, for instance, if $\delta_{n}$ is constant for each $n$ ).

Our first result provides a conceptual and easy proof of the following connection between right-weak Gibbs measures and equilibrium states.

Theorem A. Let $f: X \rightarrow X$ be a continuous map on a compact metric space $(X, d)$ whose topological entropy $h_{\text {top }}(f)$ is finite and which preserves a Borel probability measure $\mu$. Consider a continuous potential $\varphi: X \rightarrow \mathbb{R}$. If $\mu$ is a $\varphi$-right-weak Gibbs probability measure for $f$, then $\mu$ is an equilibrium state for $\varphi$.

The most used $\gamma$ in the condition (2) is $P_{\text {top }}(f, \varphi)$, though we notice that a wider choice of $\gamma$ values is receiving more attention nowadays (see, for instance, [8]). Yet, exploring further the proof of Theorem A we became aware of the most accurate choices of $\gamma$, and slightly improved [6, Proposition 3].

Theorem B. Let $f: X \rightarrow X$ be a continuous map on a compact metric space $(X, d)$ which preserves a Borel probability measure $\mu$. Consider a continuous potential $\varphi: X \rightarrow \mathbb{R}$.

(a) If $\mu$ is $(\gamma, \varphi)$-weak Gibbs for $f$, then $P_{\text {top }}(f, \varphi) \geqslant \gamma$.

(b) If $\mu$ is $(\gamma, \varphi)$-quasi Gibbs for $f$, then $P_{\text {top }}(f, \varphi)=\gamma$.

\section{A formula FOR THE LOCAL METRIC PRESSURE}

The main ingredient in the proof of Theorem $\mathrm{A}$ is a formula to estimate the local metric pressure of a continuous potential which generalizes Brin-Katok local entropy formula (cf. [2]). More precisely, having fixed a Borel $f$-invariant probability measure $\mu$ and a continuous 
potential $\varphi$, we consider the sums $S_{n}^{f} \varphi \stackrel{\text { def }}{=} \sum_{j=0}^{n-1} \varphi \circ f^{j}$ and, for $x \in X$, we define the local metric pressure of $\varphi$ at $x$ with respect to $\mu$ by

$$
P_{\mu}(x, f, \varphi)=\lim _{\varepsilon \rightarrow 0^{+}} \limsup _{n \rightarrow+\infty} \frac{-\log \mu\left(B_{n}^{f}(x, \varepsilon)\right)+S_{n}^{f} \varphi(x)}{n}
$$

if this limit exists. Note that $P_{\mu}(x, f, 0)$ evaluates the local metric entropy of $f$ at $x$ as defined by Brin and Katok. Regarding this notion, we refer the reader to [7] and [3]. (We thank the anonymous referee for calling our attention to these references.)

Proposition 2.1. Let $(X, d)$ be a compact metric space and $f: X \rightarrow X$ be a continuous transformation. Given a Borel $f$-invariant probability measure $\mu$ and a continuous potential $\varphi: X \rightarrow \mathbb{R}$, there exists a full $\mu$-measure set $\mathcal{B} \subset X$ such that the map $x \in \mathcal{B} \mapsto P_{\mu}(x, f, \varphi)$ is well defined, $\mu$-integrable, $f$-invariant and satisfies

$$
\int P_{\mu}(x, f, \varphi) d \mu=P_{\mu}(f, \varphi) .
$$

In particular, if $\mu$ is ergodic then $P_{\mu}(x, f, \varphi)=P_{\mu}(f, \varphi)$ for $\mu$-almost every $x$.

Proof. Brin-Katok's result asserts that, given a compact metric space $X$, a continuous map $f: X \rightarrow X$ and a Borel $f$-invariant probability measure $\mu$, there exists a full $\mu$-measure set $\mathcal{B K} \subset X$ such that:

(a) For every $x \in \mathcal{B K}$,

$$
\lim _{\varepsilon \rightarrow 0^{+}} \limsup _{n \rightarrow+\infty} \frac{-\log \mu\left(B_{n}^{f}(x, \varepsilon)\right)}{n}=\lim _{\varepsilon \rightarrow 0^{+}} \liminf _{n \rightarrow+\infty} \frac{-\log \mu\left(B_{n}^{f}(x, \varepsilon)\right)}{n} .
$$

(b) If $h_{\mu}(x, f)$ stands for the previous common limit, then the map $x \in \mathcal{B K} \mapsto h_{\mu}(x, f)$ is $f$-invariant and $\mu$-integrable.

(c) $\int h_{\mu}(x, f) d \mu=h_{\mu}(f)$.

Fix now a continuous potential $\varphi$ and a Borel $f$-invariant probability measure $\mu$. Birkhoff's Ergodic Theorem provides a full $\mu$-measure set $\mathcal{B}_{\varphi}$ and an $f$-invariant map

$$
x \in \mathcal{B}_{\varphi} \mapsto \widetilde{\varphi}(x) \stackrel{\text { def }}{=} \lim _{n \rightarrow+\infty} \frac{1}{n} \sum_{i=0}^{n-1} \varphi\left(f^{i}(x)\right)
$$

satisfying $\int \widetilde{\varphi} d \mu=\int \varphi d \mu$. Therefore, for every $x$ in the set $\stackrel{\mathcal{B}}{\stackrel{\text { eff }}{=}} \mathcal{B} \mathcal{K} \cap \mathcal{B}_{\varphi}$, we have

$$
\begin{aligned}
& \liminf _{n \rightarrow+\infty} \frac{-\log \mu\left(B_{n}^{f}(x, \varepsilon)\right)}{n}+\widetilde{\varphi}(x)=\liminf _{n \rightarrow+\infty} \frac{-\log \mu\left(B_{n}^{f}(x, \varepsilon)\right)+S_{n}^{f} \varphi(x)}{n} \leqslant \\
& \leqslant \limsup _{n \rightarrow+\infty} \frac{-\log \mu\left(B_{n}^{f}(x, \varepsilon)\right)+S_{n}^{f} \varphi(x)}{n}=\limsup _{n \rightarrow+\infty} \frac{-\log \mu\left(B_{n}^{f}(x, \varepsilon)\right)}{n}+\widetilde{\varphi}(x) .
\end{aligned}
$$


Taking the limit when $\varepsilon$ goes to 0 , we conclude that, for every $x \in \mathcal{B}$,

$$
\lim _{\varepsilon \rightarrow 0^{+}} \liminf _{n \rightarrow+\infty} \frac{-\log \mu\left(B_{n}^{f}(x, \varepsilon)\right)+S_{n}^{f} \varphi(x)}{n}=\lim _{\varepsilon \rightarrow 0^{+}} \limsup _{n \rightarrow+\infty} \frac{-\log \mu\left(B_{n}^{f}(x, \varepsilon)\right)+S_{n}^{f} \varphi(x)}{n} .
$$

So $P_{\mu}(x, f, \varphi)$ is well defined, $f$-invariant, $\mu$-integrable and

$$
P_{\mu}(x, f, \varphi)=h_{\mu}(x, f)+\widetilde{\varphi}(x)
$$

at $\mu$ almost every $x \in X$. Using the previous item (c) of Brin-Katok's result, we obtain

$$
\int P_{\mu}(x, f, \varphi) d \mu=\int\left[h_{\mu}(x, f)+\widetilde{\varphi}(x)\right] d \mu=h_{\mu}(f)+\int \varphi d \mu
$$

as claimed. To complete the proof of Proposition 2.1, we are left to remark that, if $\mu$ is ergodic, then the $f$-invariant map $x \mapsto P_{\mu}(x, f, \varphi)$ is constant in the set $\mathcal{B}$, and so $P_{\mu}(x, f, \varphi)=$ $h_{\mu}(f)+\int \varphi d \mu$ at $\mu$ almost every $x \in X$.

\section{Proof of Theorem A}

Firstly recall that, given a compact metric space $X$ and a continuous map $f: X \rightarrow X$, the pressure map $P_{\text {top }}(f,):. C^{0}(X, \mathbb{R}) \rightarrow \mathbb{R} \cup\{+\infty\}$, defined on the space $C^{0}(X, \mathbb{R})$ of continuous potentials, is either finite valued or constantly $+\infty$ (cf. [9, §9.2]).

Consider a continuous potential $\varphi$ and a $\varphi$-right-weak Gibbs measure $\mu$ for the dynamics $f$, and gather the corresponding $\varepsilon_{0}, \Lambda$ and $\left(\delta_{n}(\varepsilon, x)\right)_{n \in \mathbb{N}}$ satisfying equation (1) and the right inequality of (2) for every $x \in \Lambda$ and every $n \in \mathbb{N}$ (or every large enough $n$ ), that is

$$
\frac{\mu\left(B_{n}^{f}(x, \varepsilon)\right)}{\exp \left(-n P_{\text {top }}(f, \varphi)+S_{n}^{f} \varphi(x)\right)} \leqslant \delta_{n}(\varepsilon, x) .
$$

As we are assuming that $h_{\mathrm{top}}(f)<+\infty$, we know that $P_{\text {top }}(f, \varphi)$ is finite. Rewriting (4), we obtain

$$
P_{\text {top }}(f, \varphi)-\frac{\log \delta_{n}(\varepsilon, x)}{n} \leqslant \frac{-\log \mu\left(B_{n}^{f}(x, \varepsilon)\right)+S_{n}^{f} \varphi(x)}{n} .
$$

For every $x \in \mathcal{B} \mathcal{K} \cap \mathcal{B}_{\varphi} \cap \Lambda$, taking $\lim \sup _{n \rightarrow+\infty}$ (or $\liminf _{n \rightarrow+\infty}$ ) and afterwards evaluating the limit of the last inequality as $\varepsilon$ goes to $0^{+}$, we get

$$
P_{\mu}(x, f, \varphi) \geqslant P_{\text {top }}(f, \varphi) \quad \text { at } \mu \text {-a.e. } x \in X \text {. }
$$

Thus, applying Proposition 2.1, we conclude that

$$
h_{\mu}(f)+\int \varphi d \mu=\int P_{\mu}(x, f, \varphi) d \mu \geqslant \int P_{\text {top }}(f, \varphi) d \mu=P_{\text {top }}(f, \varphi) .
$$

Therefore $\mu$ is an equilibrium state for $\varphi$. 


\section{Proof of Theorem B}

With a simple computation we turn the inequalities (2), for every $x \in \mathcal{B} \mathcal{K} \cap \mathcal{B}_{\varphi} \cap \Lambda$, into

$$
\gamma-\frac{\log \delta_{n}(\varepsilon, x)}{n} \leqslant \frac{-\log \mu\left(B_{n}^{f}(x, \varepsilon)\right)+S_{n}^{f} \varphi(x)}{n} \leqslant \gamma-\frac{\log \delta_{n}(\varepsilon, x)^{-1}}{n} .
$$

Since $\mu$ is $f$-invariant, taking $\lim \sup _{n \rightarrow+\infty}\left(\right.$ or $\left.\lim \inf _{n \rightarrow+\infty}\right)$ and afterwards the limit as $\varepsilon$ goes to 0 , Proposition 2.1 yields

$$
P_{\mu}(x, f, \varphi)=\gamma \quad \text { at } \mu-\text { a.e. } x \in X
$$

and

$$
h_{\mu}(f)+\int \varphi d \mu=\int P_{\mu}(x, f, \varphi) d \mu=\gamma .
$$

Therefore, $P_{\text {top }}(f, \varphi) \geqslant \gamma$.

We are left to show that $P_{\text {top }}(f, \varphi) \leqslant \gamma$ if $\mu$ is $(\gamma, \varphi)$-quasi Gibbs. We start observing that $\mu$ is $(\gamma, \varphi)$-quasi Gibbs if only if it is $(0, \varphi-\gamma)$-quasi Gibbs. Moreover:

Lemma 4.1. Given a continuous potential $\psi$, if $f$ admits a $(0, \psi)$-quasi Gibbs probability measure, then $P_{\text {top }}(f, \psi) \leqslant 0$.

Proof. If the potential $\psi$ is Hölder continuous, then the lemma is a consequence of the following assertion, whose proof is a straightforward adaptation of Bowen's argument to show Proposition 4.8 (a) of [1].

Claim: If $v$ is a $(0, \psi)$-left-quasi Gibbs probability measure for $f$ and a Hölder continuous potential $\psi$, then there exists $\varepsilon_{0}>0$ such that, for every $0<\varepsilon<\varepsilon_{0}$, one has

$$
P_{\text {top }}(f, \psi) \leqslant \liminf _{n \rightarrow+\infty} \frac{1}{n} \log v\left(B_{n}^{f}(\varepsilon)\right)=0
$$

where $B_{n}^{f}(\varepsilon)$ stands for the union $\bigcup_{x \in X} B_{n}^{f}(x, \varepsilon)$.

Regarding more general potentials, the lemma is a particular case of [5, Proposition 3.1], whose proof simply requires a further adjustment of Bowen's reasoning. This proposition ensures that:

Claim: If $v$ is a $(0, \psi)$-left-quasi Gibbs probability measure for $f$ and a continuous potential $\psi$, then for every $f$-invariant ergodic Borel probability measure $\theta$ on $X$ one has

$$
h_{\theta}(f)+\int \psi d \theta \leqslant 0 .
$$

Consequently, by the Variational Principle,

$$
P_{\text {top }}(f, \psi)=\sup _{\{f \text {-invariant ergodic } \theta\}}\left\{h_{\theta}(f)+\int \psi d \theta\right\} \leqslant 0 .
$$


We are ready to complete the proof of Theorem B since, by Lemma 4.1,

$$
P_{\text {top }}(f, \varphi)=P_{\text {top }}(f, \varphi-\gamma)+\gamma \leqslant \gamma .
$$

In particular, due to (5), $\mu$ is an equilibrium state for $\varphi$.

\section{Non-inVariant Gibbs measures}

As far as we know it is still an open question under what additional conditions, other than invariance, is a weak Gibbs measure either an equilibrium state (even if for another potential somehow related to the original one) or equivalent to an equilibrium state. Considering a stronger definition of the Gibbs property and assuming that the dynamical system is a homeomorphism satisfying expansiveness and specification, the answer to this question is positive (cf. [4]). We may also ask whether the presence of a Gibbs measure for some potential prompts the existence of an equilibrium state for that potential.

The next three examples evince the distinction between being a Gibbs measure, an invariant measure, an equilibrium state or else equivalent to an equilibrium state.

Example 5.1. In the context of Ruelle-expanding maps, the transfer operator provides Gibbs measures which are not invariant. So they are not equilibrium states, despite being equivalent to them (cf. [1]).

Example 5.2. While constructing equilibrium states for Hölder potentials on basic sets of $C^{2}$ diffeomorphisms acting on Riemannian manifolds (cf. [1]), Bowen shows a Volume Lemma which asserts that the volume measure on the manifold is a $\left(0, \varphi^{u}\right)$-Gibbs measure, where $\varphi^{u}=-\log \operatorname{Jac}_{\mathrm{Df}_{\mid}}$, though it may be non-invariant and not equivalent to the equilibrium state of $\varphi^{u}$.

Example 5.3. Consider a Markov unilateral chain $\Sigma_{A}^{+} \subset\{0,1\}^{\mathbb{N}}$ associated to an irreducible 2-dimensional matrix $A$. The measure of maximal entropy $\mu$ of the shift map acting on $\Sigma_{A}^{+}$, which is the equilibrium state of $\varphi \equiv 0$, is a 0 -Gibbs measure (cf. [1, Theorem 1.16]) determined by a probability vector $\left(p_{0}, p_{1}\right)$ which is fixed under the action of a stochastic matrix $P=\left(p_{i j}\right)_{i, j \in\{0,1\}}$ (cf. [9, Theorem 8.10]), that is, $p P=p$. Accordingly, the $\mu$-measure of a cylinder

$$
\mathcal{C}\left(k ; a_{0}, a_{1}, \cdots, a_{n}\right):=\left\{\left(x_{n}\right)_{n \in \mathbb{N}} \in \Sigma_{A}^{+}: x_{k}=a_{0}, \cdots, x_{n+k}=a_{n}\right\}
$$

where $k, n \in \mathbb{N}$ and $a_{j} \in\{0,1\}$ for every $0 \leqslant j \leqslant n$, is given by $p_{a_{0}} p_{a_{0} a_{1}} \cdots p_{a_{n-1} a_{n}}$. Take now a probability vector $\left(q_{0}, q_{1}\right)$ such that $q P \neq q$. Then the corresponding $v$ is not $\sigma$ invariant, though it is a Markov measure and 0-Gibbs as well. Indeed, for every cylinder $\mathcal{C}\left(k ; a_{0}, a_{1}, \cdots, a_{n}\right)$, one has

$$
v\left(\mathcal{C}\left(k ; a_{0}, a_{1}, \cdots, a_{n}\right)\right)=q_{a_{0}} p_{a_{0} a_{1}} \cdots p_{a_{n-1} a_{n}}=\left(\frac{q_{a_{0}}}{p_{a_{0}}}\right) p_{a_{0}} p_{a_{0} a_{1}} \cdots p_{a_{n-1} a_{n}}
$$

and so

$$
\alpha \mu\left(\mathrm{C}\left(k ; a_{0}, a_{1}, \cdots, a_{n}\right)\right) \leqslant v\left(\mathrm{C}\left(k ; a_{0}, a_{1}, \cdots, a_{n}\right)\right) \leqslant \beta \mu\left(\mathrm{C}\left(k ; a_{0}, a_{1}, \cdots, a_{n}\right)\right)
$$


where $\alpha=\min _{0 \leqslant j \leqslant 1}\left\{\frac{q_{j}}{p_{j}}\right\}>0$ and $\beta=\max _{0 \leqslant j \leqslant 1}\left\{\frac{q_{j}}{p_{j}}\right\}>0$.

\section{RefERENCES}

[1] R. Bowen. Equilibrium States and the Ergodic Theory of Anosov Diffeomorphisms. Lecture Notes in Math. 470, Springer-Verlag Berlin Heidelberg, 1975.

[2] M. Brin and A. Katok. On local entropy. Geometric Dynamics (Rio de Janeiro), Springer-Verlag Berlin,1981.

[3] Y. Cao, H. Hu and Y. Zhao. Nonadditive measure-theoretic pressure and applications to dimensions of an ergodic measure. Ergodic Theory \& Dynam. Systems 33:3 (2013) 831-850.

[4] N. Haydn and D. Ruelle. Equivalence of Gibbs and equilibrium states for homeomorphisms satisfying expansiveness and specification. Commun. Math. Phys. 148 (1992) 155-167.

[5] C.-E. Pfister and W. Sullivan. Large deviations estimates for dynamical systems without the specification property. Application to the $\beta$-shifts. Nonlinearity 18 (2005) 237-261.

[6] C.-E. Pfister and W. Sullivan. Weak Gibbs measures and large deviations. Nonlinearity 31 (2018) 49-53.

[7] X. Tang, W. Cheng and Y. Zhao. Variational principle for topological pressures on subsets. J. Math. Anal. Appl. 424:2 (2015) 1272-1285.

[8] P. Varandas. Non-uniform specification and large deviations for weak Gibbs measures J. Stat. Phys. 146:2 (2012) 330-358.

[9] P. Walters. An Introduction to Ergodic Theory. Graduate Texts in Mathematics 79, Springer-Verlag New York, 1981.

Maria Carvalho, Centro de Matemática da Universidade do Porto, Rua do Campo Alegre 687, 4169-007 Porto, Portugal

E-mail address: mpcarval@fc.up.pt

Sebastián A. Pérez, Centro de Matemática da Universidade do Porto, Rua do Campo Alegre 687, 4169-007 Porto, Portugal

E-mail address: sebastian.opazo@fc.up.pt 